\title{
Improving Routing Performances to Provide Internet Connectivity in VANETs over IEEE 802.11p
}

\author{
Driss ABADA \\ Laboratory LABSIV \\ Faculty of sciences \\ Agadir, Morocco
}

\author{
Abdellah MASSAQ \\ Laboratory OSCARS \\ National School of Applied sciences \\ Marrakech, Morocco
}

\author{
Abdellah BOULOUZ \\ Laboratory LABSIV \\ Faculty of sciences \\ Agadir, Morocco
}

\begin{abstract}
In the intelligent transportation systems, many applications and services could be offered in the road via Internet. Providing these applications over vehicular ad hoc network (VANETs) technology may require good performances in routing. The channel fading and quality of received signal are the two main factors which affect the Mobile ad hoc network performances as well as mobility of vehicles, in terms of throughput and delay packets that are relevant to the performance evaluation of the routing protocols. In this paper, we propose an efficient relay selection scheme based on Contention Based Forwarding (CBF) and Fuzzy Logic System (FLS) that considers two important Quality of Service parameters such as link stability, and quality of received signal to select a potential relay vehicle, in order to improve the routing performances in the network. The simulation results show that the proposed relay selection scheme enhances throughput, and decreases packet delay and overhead comparing it with an existing link stability based routing protocol(MBRP) and M-AODV+.
\end{abstract} ity

Keywords-VANET; routing; link stability; fading; RSS; mobil-

\section{INTRODUCTION}

Several wireless networking technologies such as WiMAX [1], 3G/4G/5G cellular networks [2], Long Term Evolution [3], [4], IEEE 802.11-based vehicular ad hoc networks (VANETs) named IEEE $802.11 \mathrm{p}$, are available actually to achieve Internet connectivity for VANETs. IEEE 802.11p [5], also known as Wireless Access in Vehicular Environment (WAVE) protocol is an enhancement to the 802.11 physical layer (PHY) and medium access control (MAC) to support high vehicular mobility and faster topological changes. This standard is used as groundwork for Dedicated Short Range Communication (DSRC). It operates in the $5.9 \mathrm{GHz}$ band and supports both vehicle-to-vehicle (V2V) and vehicle-toinfrastructure (V2I) communications. The maximum data rate supported by this standard is $27 \mathrm{Mbps}$. The mobility supported is up to $200 \mathrm{kmph}$, making this suitable for VANETs applications involving highway scenario. IEEE $802.11 \mathrm{p}$ provides a short-range radio communication of approximately $300 \mathrm{~m}$.

Vehicular ad hoc networks (VANETs) are characterized by frequent and fast topology changes, which are introduced by vehicles mobility as well as by channel fading. Link stability is often measured using mobility parameters (position, speed, direction, etc) and the impact of fading channel on is disregarded. In this paper, we have combined the impact of vehicles mobility and fading channel statistics on link stability. Moreover, we have improved relay selection scheme by considering the link stability and quality of received signal, in order to make vehicles able to select the most stable with the minimum fading, and the strongest received signal route from them to the road side units (RSUs) which are part of wired network (Internet). Hop count-based routing protocols; select the shortest path length in term of a number of hops. However, these protocols do not typically select a route with sufficient lifetime to maintain the longest possible duration of communication with RSUs, which make existing routing protocols basically designed for MANETs not suitable for VANETs. For example M-AODV+ [6] which is an extension version of AODV+ [6] proposed to support the reliability of V2V communication in VANETs by enabling V2I and I2I communications as alternative communication links among vehicles when single hop or multi-hop communication in the network is not possible. In contrast, many routing protocols in the literature such as [7], [8], [9], are proposed to utilize a metric characterizing link stability to choose the most stable route in the network.

Communications in vehicular networks are carried out in an unfavorable external environment for the establishment of radio links, because of the multitude of obstacles (forests, mountains, buildings ...), especially in urban areas. These obstacles cause a severe degradation in the quality of the signals transmission. In the one side, Multipath fading introduced will have an impact on the stability of link as well as mobility of nodes and consequently on the network performances. On the other side, received signal fluctuations will certainly increase the route failures and dropped packets in the network.

In this paper, we have modified relay selection scheme proposed in [10] which base on the contention-based forwarding scheme, to take into account to channel fading and the quality of received signal of a link. We have integrated into the relay selection scheme, two important features: link stability and link quality in term of received signal. Link stability is measured in function of mobility parameters and fading statistics. However, quality of link received signal is measured using a fuzzy logic system between received signal strength (RSS) and mobility.

The layout of this paper is as follows. In Section II, we present the related work. Our proposed work is detailed in Section III. The performance of our protocol is discussed in Section IV. Finally, we give the conclusion and future work directions in Section V. 


\section{RELATED WORK}

In the paper [11], the other has proposed an efficient relay selection mechanism which considers the link stability as the main routing metric to select a potential relay. The link stability is measured not only in function of the mobility parameters such as geographic location, direction, and speed of vehicles but also in function of channel fading statistics. In congested scenarios, basing only on this feature of stability, one vehicle may be selected as a relay for multiple vehicles and if the number of relay requests exceeds the service capacity of this vehicle, it might get overloaded. Thus, the dropping packets and large contention delay in the MAC layer may be happening in the network. As the solution, the authors proposed a new mechanism to adapt the waiting time of each node according to its channel access availability and its queue occupancy using the fuzzy logic system. In this work, the quality of received signal is not taking into consideration which may degrade the network performances especially in the more realistic environment.

MBRP (Mobility Based Routing Protocol) [10] is an efficient routing protocol for connecting vehicular networks to the Internet which uses the characteristics of vehicle movements to predict the future behavior of vehicles, and to select a route with the longest lifetime. The proposed protocol aims to broadcast the advertisement messages through multi-hops in the predefined geographic zone and uses a distributed manner to select relay for a re-broadcasting message, this approach will connect VANETs to Internet on minimizing overhead without flooding network through most stable route. MBRP does not consider the fading feature of wireless channels and the received signal quality.

In this work [12], the authors proposed a multilayer cooperation framework to offer passengers in vehicle continuous and best available access to the Internet with a controlled Quality of Service (QoS). This approach exploits the presence of Road Side Unite installed along the route acting as a gateway to the wired network (Internet), some vehicles acting as a mobile gateway for other vehicles with just a short-range radio interface. This framework consists of estimating in the MAC layer some parameters (expected delay and available throughput) and using them in the network layer, in order to select the route with the minimum bit rate requirement and the residual delay allowed to be spent from the gateway to the destination. At the PHY layer, each node in VANET measures periodically, the received signal strength indicator (RSSI) on each active link to their neighbors, in order, to select the most stable route from vehicle to the gateway. In this framework, vehicles base on the reactive approach to building QoS network paths to the gateway, the disadvantages is that reactive route discovery increase end to end delay. The centralized method (where nodes broadcast periodically hello message) which used to measure RSSI in order to estimate link lifetime, is not suitable for VANET because it causes flooding and broadcast storms in the network which will increase a bandwidth consummation and decrease data throughput.

\section{PROPOSED WORK}

\section{A. Channel Model of Vehicular Ad hoc Network}

Nakagami-m distribution seems to be most suitable to modeled communication in VANET networks in the absence of interferences [13], [14]. In this paper, we assume that the communication between nodes in VANETs is doing over Nakagami fading channel and we aim to select a most stable path with minimum fading. The wireless channel model includes the effects of small-scale fading and large-scale path loss. Here we assume that every node has the same transmission power and that the transmission power is constant. For a transmission over a distance $d$, in the presence of Nakagami$\mathrm{m}$ fading, instantaneous received signal power $p$ is Gamma distributed. According to [13], in the fast fading $(m=3)$, the probability that a packet is successfully received in the absence of interference, is deduced from the probability that the packet's received signal is stronger than the reception threshold, which represents the minimum acceptable value of received power, that is,

$\Psi=P_{r}\left(p \geq R_{t h}\right)=\exp \left(-3 \cdot \frac{R_{t h}}{p}\right) \cdot\left(1+3 \cdot \frac{R_{t h}}{p}+\frac{9}{2} \cdot\left(\frac{R_{t h}}{p}\right)^{2}\right)$

\section{B. Mobility and Fading based Link Stability Metric Estimation}

In this paper, the link stability is estimated using a routing metric called Effective Link Operation Duration (ELOD) proposed in [15]. The authors defined it as the duration within the link lifetime in which the received signal power above an acceptable threshold $R_{t h}$. To estimate the ELOD first, the author [15] used mobility parameters to predict the link lifetime and then combine the link lifetime with the fading channel statistics. Noted that the authors [15] have used the Rayleigh distribution as propagation model to model the fading. However, the fading with Rayleigh model is not more satiable in VANET environment, because, it assumes that all the transmitted signals arriving at the receiver are allocated in the same power.

The effective link operation duration between two vehicles $i$ and $j$ noted $E L O D_{i j}$ can be estimated as in [15] as follow:

$$
E L O D_{i j}=\operatorname{Let}_{i j} \times E[\Psi]
$$

where Let $_{i j}$ is a lifetime of link formed between vehicles $i$ and $j$ which are positioned at locations $\left(x_{i}, y_{i}\right),\left(x_{j}, y_{j}\right)$, and move in direction $\theta_{i}, \theta_{j}$ with speed $v_{i}, v_{j}$ respectively. Assuming that all vehicles have the same transmission range $R$, the link expiration time noted Let $_{i j}$ can be estimated according to [10] using mobility parameters as follows,

$$
\text { Let }_{i j}=\frac{\sqrt{\left(a^{2}+c^{2}\right) R^{2}-(a d-b c)^{2}}-(a b+c d)}{a^{2}+c^{2}}
$$

where,

$a=v_{i} \cos \theta_{i}-v_{j} \cos \theta_{j}$

$b=x_{i}-x_{j}$

$c=v_{i} \sin \theta_{i}-v_{j} \sin \theta_{j}$

$d=y_{i}-y_{j}$.

and $\Psi$ represents the probability of a link between vehicle $i$ and vehicle $j$ which are in connection within link lifetime in 


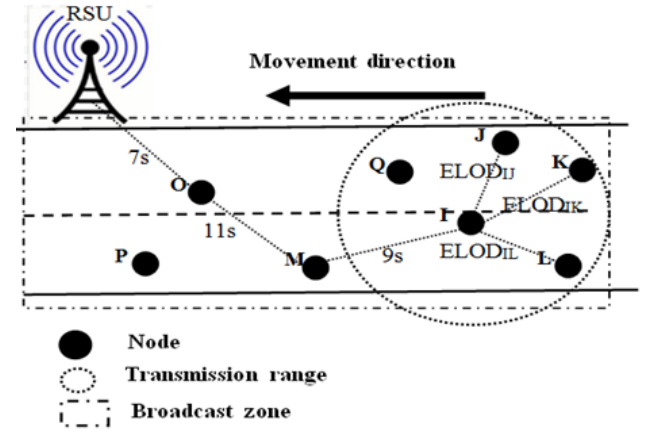

Fig. 1. Route selection based on EROD illustration

which the received signal power is above a certain predefined threshold. $E[\Psi]$ is the expected value of probability $\Psi$.

Now, let $R_{N-1}$ the route from the RSU to the source vehicle, and in each route there are $N-1$ links between $N$ vehicles. The effective route operation duration noted $E R O D_{N-1}$ is defined to be the shortest lifetime along the route $R_{N-1}$.

$$
E R O D_{N-1}=M_{i n}^{N} n_{i=1, j=i+1} E L O D_{i j}
$$

As illustrated in Fig. 1, the vehicles $\mathrm{O}$ and $\mathrm{M}$ are selected as relays to form the route from RSU to vehicle I. The ELOD of each link is written over the link. EROD is its effective route operation duration which equals $7 \mathrm{~s}$ in this case. The vehicle I in this example is selected as a next relay and will rebroadcast the message to its neighbors such as J, K and L. Noted that the message is disseminated in the opposite direction of vehicles movement, and all vehicles move in the same direction.

The model in [13] neglects large scale fading (shadowing) and considers a quadratic path-loss according to the Friis model $(n=2)$ [16]. Therefore, in the absence of interferences, the average reception power $p$ at a distance $\mathrm{d}$, and the reception threshold $R_{t h}$ are :

$$
p \infty d^{-2}
$$

and

$$
R_{t h} \infty R^{-2}
$$

The model calculates the expected probability of successfully receiving a message at distance $d$ given transmission range $R$ in the absence of simultaneous transmissions. The packet reception probability formula (1) is reformed as follows:

$$
\Psi=\exp \left(-3 \cdot\left(\frac{d}{R}\right)^{2}\right) \cdot\left(1+3 \cdot\left(\frac{d}{R}\right)^{2}+\frac{9}{2} \cdot\left(\frac{d}{R}\right)^{4}\right)
$$

Due to the mobility of nodes, the relative distance $d$ varies at the times, consequently, the probability $\Psi$ varies with node movement. To account for this random variation, we replace $d$ in (7) with a continuous random variable $Z$, which represents the distance between the sender and the receiver. As mentioned previously, using the probability that a link is not in a fade, we can estimate the link operation duration. According to [15], the expected value of the probability $\Psi$ can be determined as follow:

$$
E[\Psi]=\int_{d_{\min }}^{R} \Psi(z) f_{Z}(z) d z
$$

where $f_{Z}(z)$ is the probability density function (pdf). According to the expression of the function $f_{Z}$ defined in [15], we distinguished tree cases of expected value $E[\Psi]$ :

- $\quad$ Case $1:$ If the relative distance between vehicles $i$ and $j$ remains constant during their movement, the average $E[\Psi]$ for the link during the prediction period is

$$
E[\psi]=\exp \left(-3 \cdot\left(\frac{d_{i j}}{R}\right)^{2}\right) \cdot\left(1+3 \cdot\left(\frac{d_{i j}}{R}\right)^{2}+\frac{9}{2} \cdot\left(\frac{d_{i j}}{R}\right)^{4}\right)
$$

- Case 2: If the two vehicles only move away from each other during the prediction period, the pdf of $Z$ is

$$
f_{Z}(z)= \begin{cases}0 & \text { if } z<d_{i j} \\ \frac{1}{R-d_{i j}} & \text { if } d_{i j} \leq z \leq R \\ 0 & \text { if } z>R\end{cases}
$$

The expression of expected value $E[\Psi]$ is :

$$
\begin{aligned}
& E[\Psi]=\frac{1}{R-d_{i j}} \int_{d_{i j}}^{R} \exp \left(-3 \cdot\left(\frac{z}{R}\right)^{2}\right) . \\
& \left(1+3 \cdot\left(\frac{z}{R}\right)^{2}+\frac{9}{2} \cdot\left(\frac{z}{R}\right)^{4}\right) d z
\end{aligned}
$$

- Case 3: If the two vehicles first move toward each other, then apart some time later, the pdf of $Z$ is

$$
f_{Z}(z)= \begin{cases}\frac{2}{R+d_{i j}} & \text { if } z<d_{i j} \\ \frac{1}{R+d_{i j}} & \text { if } d_{i j} \leq z \leq R \\ 0 & \text { if } z>R\end{cases}
$$

The expression of expected value $E[\Psi]$ is :

$$
\begin{aligned}
& E[\Psi]= \frac{2}{R+d_{i j}} \int_{0}^{d_{i j}} \exp \left(-3 \cdot\left(\frac{z}{R}\right)^{2}\right) \cdot\left(1+3 \cdot\left(\frac{z}{R}\right)^{2}+\right. \\
&\left.\frac{9}{2} \cdot\left(\frac{z}{R}\right)^{4}\right) d z+\frac{1}{R+d_{i j}} \int_{d_{i j}}^{R} \exp \left(-3 \cdot\left(\frac{z}{R}\right)^{2}\right) . \\
&\left(1+3 \cdot\left(\frac{z}{R}\right)^{2}+\frac{9}{2} \cdot\left(\frac{z}{R}\right)^{4}\right) d z
\end{aligned}
$$

where $d_{i j}=\sqrt{\left(x_{i}-x_{j}\right)^{2}+\left(y_{i}-y_{j}\right)^{2}}$.

\section{Design of Fuzzy Inference based Mobility and RSS}

As it is explained previously, our relay selection mechanism aims to improve relay selection by considering received signal strength (RSS). The reason behind choosing RSS is to ensure that the packets will be received with enough reception power strength, reduce selecting failures routes and increase the reliability of them. The relative speed between sender and receiver is a metric among others which has a good consistency with RSS. The faster source vehicle moves towards to receiver, the faster will be the increase in the link RSS. Similarly, the faster the source vehicle moves away from the receiver, the faster will be the decline in the link RSS. For this purpose, we take advantage of the fuzzy logic system (FLS) which received signal strength and mobility speed factors are the fuzzy inputs and quality of link received signal (QLRS) is fuzzy output. More details of FLS are given to papers [17], [18].

Upon reception of advertisement message from previous relay (RSU or relay vehicle), each vehicle measures RSS of packet and calculates RSS factor (RSSF) and mobility factor (MF) as shown in the following formulas:

$$
R S S F=1-\frac{R S S_{\text {thresh }}}{R S S}
$$




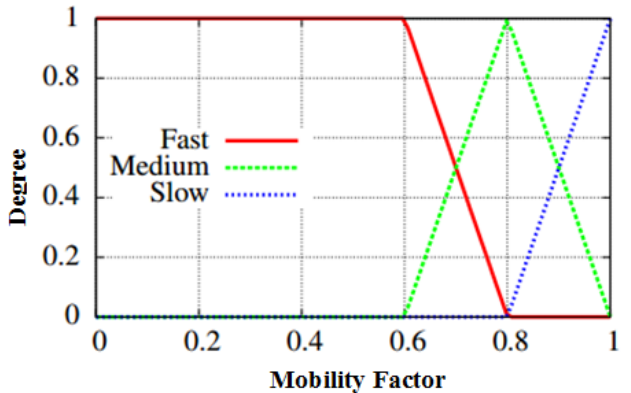

Fig. 2. Mobility membership function

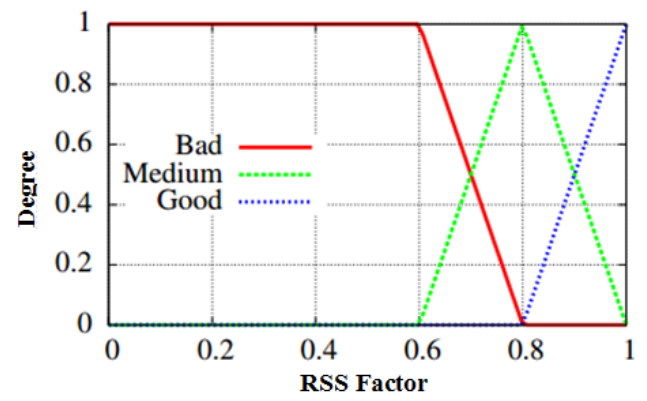

Fig. 3. RSS membership function

$$
M F=1-\frac{\left|V_{r}-V_{s}\right|}{V_{\max }}
$$

where $R S S_{\text {thresh }}$ is the received signal strength threshold, $V_{r}$ and $V_{s}$ are the receiver and source speeds respectively and $V_{\max }$ is the maximum speed in the network. We assume that vehicles are all equipped with GPS devices and keep their speed during prediction link lifetime. The input fuzzy variables of $R S S F$ and $M F$ are classified into three levels. This grouping strategy gives more clues on the weakness and strength of input variables and helps to generate more accurate output data.

Upon reception of advertisement message, each vehicle uses the fuzzy system to calculate the value of the received signal quality of link formed with the sender, based on mobility, moving direction (the sender and the receiver moving direction is towards or away) and the RSS. For each receiver vehicle in competition to be relay, the steps for calculating the numerical value of QLRS are as follows:

- Fuzzification

Fuzzification is the process of converting a numeric value to a fuzzy value by using a predefined membership function. The predefined linguistic variables and membership functions are used to convert the mobility factor and the RSS factor (taking into account the moving direction) of corresponding fuzzy values. The linguistic variables defined for mobility are Slow, Medium, Fast, and for RSS are Good, Medium, Bad. Fig. 2 and Fig. 3 show the membership function of mobility factor and RSS factor respectively.

- Rules base

This step consists of mapping the fuzzy values to the predefined IF/THEN rules and then combining
TABLE I. FUZZY RULES

\begin{tabular}{|c||c||c||c||c|}
\hline & IF & & & THEN \\
\hline Rules & Mobility & RSS & Moving direction & QLRS \\
\hline R1 & Slow & Good & Towards & Very good \\
\hline R2 & Medium & Good & Towards & Good \\
\hline R3 & Fast & Good & Towards & Medium \\
\hline R4 & Slow & Medium & Towards & Good \\
\hline R5 & Medium & Medium & Towards & Medium \\
\hline R6 & Fast & Medium & Towards & Low \\
\hline R7 & Slow & Bad & Towards & Low \\
\hline R8 & Medium & Bad & Towards & Very Low \\
\hline R9 & Fast & Bad & Towards & Not acceptable \\
\hline R10 & Slow & Good & Away & Good \\
\hline R11 & Medium & Good & Away & Medium \\
\hline R12 & Fast & Good & Away & Low \\
\hline R13 & Slow & Medium & Away & Medium \\
\hline R14 & Medium & Medium & Away & Low \\
\hline R15 & Fast & Medium & Away & Very low \\
\hline R16 & Slow & Bad & Away & Very Low \\
\hline R17 & Medium & Bad & Away & Not acceptable \\
\hline R18 & Fast & Bad & Away & Not acceptable \\
\hline & & & & \\
\hline
\end{tabular}

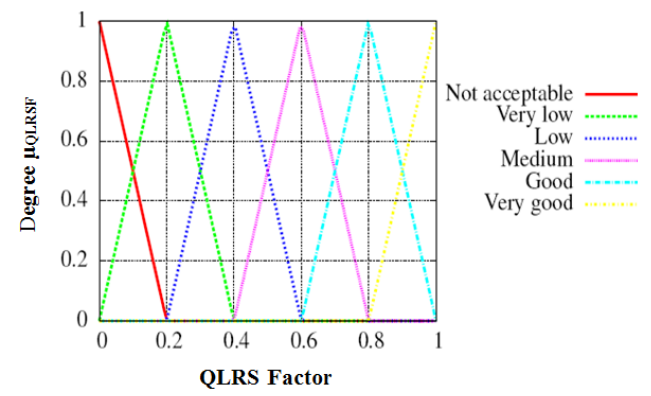

Fig. 4. Quality of link received signal membership function

them to get the fuzzy value of the quality of link received signal (QLRS) as the output value. Based on the fuzzy values of mobility factor and the RSS factor, the receiver uses the IF/ THEN rules defined in Tables I to calculate linguistic values of QLRS. The linguistic values of the QLRS are Very good, Good, Medium, Bad, Very Bad, Not acceptable.

- Defuzzification

Defuzzification is the process of converting the output fuzzy value to numerical value based on output membership function and corresponding membership degrees. The fuzzy membership function of QLRS is defined in Fig. 4.

There are various methods used for defuzzifying the fuzzy result. In this work, we have used a weighted average method to calculate the numerical value of $Q L R S$ noted $N Q L R S$ as follows:

$$
N Q L R S=\frac{\sum x \cdot \mu_{Q L R S F}}{\sum \mu_{Q L R S F}}
$$

where $\mu_{Q L R S F}$ is a degree corresponding to membership function. represents the corresponding percentage of each value obtained of fuzzy output variable QLRS. The vehicle having a maximum value of NQLRS will have a better chance to be relay. 


\section{Relay Selection Scheme}

There are three main types of VANET routing protocols: topology-based routing, position-based forwarding, and contention-based forwarding [10], [19]. Many recent works [20], [21], [22] demonstrate that contention-based forwarding (CBF) approach is preferable in highly dynamic vehicular environments. In this paper, the selection of next hop is performed by means of contention, by improving the $\mathrm{CBF}$ approach.

Our relay selection scheme is an enhancement of modified contention-based forwarding approach in [10], the relay should be the node that has the most stable and the lowest fading link with the source of the message (RSU or previous relay). Furthermore, we are also interested in the received signal strength of message which lies in its better consistency compared to the relative speed between source vehicle and receiver.

Note $L s$ the link stability function which depends to effective link operation duration ELOD according to the expression

$$
L s=1-\exp \left(\frac{-E L O D}{\lambda}\right)
$$

where $\lambda$ is a constant that defines the rate at which the function is rising.

In the equation 17 proposed in [10], we have replaced link expiration time (Let) with ELOD in order to take into consideration the impact of mobility and fading on the link stability. The result of this function $L s$ is used to fixed the waiting time of each vehicle contending to be relay.

Considering ELOD as a routing parameter, the multi-hop communication will be performed through the most stable route. Consequently, the network performances will be enhanced. However, routing data packets via a route which contains an important number of hops in the ad hoc network may experience large medium access contention, interference, congestion, and packet collisions, because of shared channel among neighboring nodes. Therefore, it will be important to add another metric alongside link stability in the relay selection, in order to select the most stable route with the minimum number of hops. For this purpose, we have combined the link stability function $L s$ with another metric proposed in [10], named Progress feature $(P f)$ which allows according to the authors to select the shortest path in term of hops. The expression of $P F$ is :

$$
P F=\frac{\cos \left(\theta_{i}-\theta_{j}\right) \times d_{i j}}{R}
$$

where $d_{i j}$ is the distance between vehicle $i$ (sender), and vehicle $j$ (current receiver), and $R$ is the transmission range of vehicles. $\theta_{i}$ and $\theta_{j}$ are respectively the direction angles of vehicles. North axis is used by vehicles as the reference for the direction angle.

The two relay selection metrics are combined in the global function $F$ using weighted mean. The function $F$ is defined as follows :

$$
F=k \times L s+(1-k) \times P F
$$

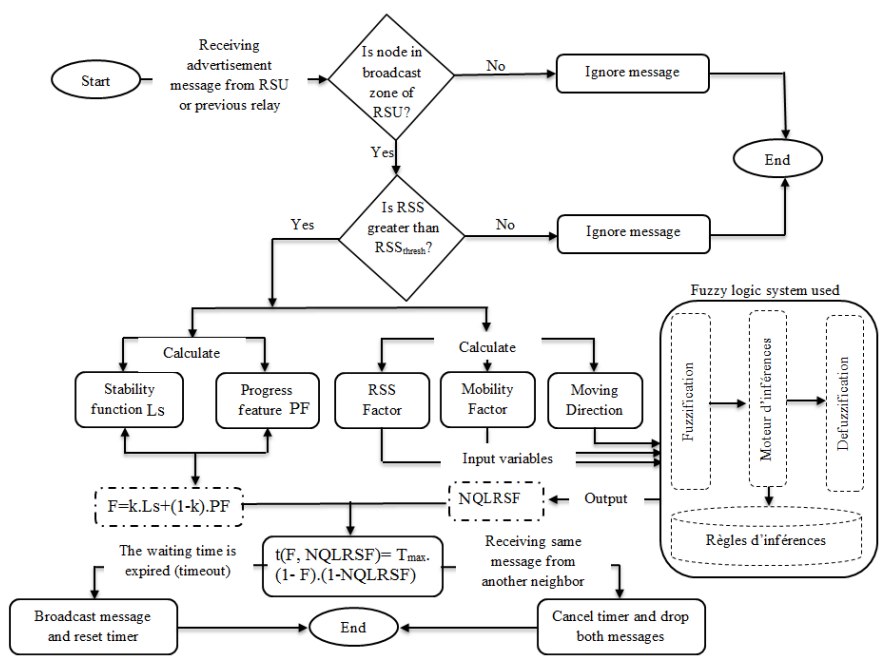

Fig. 5. Flowchart of proposed relay selection scheme

where $k$ may be selected in $\left[\frac{1}{2}, 1\right]$ to give more weight for $L s$ than PF. That means the forwarding metric with higher weighting factor which is link stability has more impact on the contention based forwarding process. For the contention over link stability and furthest neighbor,the waiting timer is:

$$
t(F)=T_{\max } \times(1-F)
$$

where $T_{\max }$ is a maximum forwarding time.

Another parameter which is not less important than the first is taken into account in our relay selection scheme, it is the factor of the quality of link received signal calculated according to the mobility and RSS using the fuzzy system (see the previous section). In order to consider the quality of link received signal in the relay selection, we reform our definition of equation 20 as follows:

$$
t(F, N Q L R S)=T_{\max } \times(1-F) \times(1-N Q L R S)
$$

where $N Q L R S$ is a numerical value of $Q L R S$ calculated using fuzzy system, and $T_{\max }$ is the maximum time that the contending node waits before rebroadcasting.

The Fig. 5 presents a flowchart of a proposed relay selection scheme based on CBF and FLS.

\section{E. Route to RSU Descovery}

The process of route discovery is similar to that proposed in [11]. In this work, we have adopted a hybrid approach which combines the proactive and reactive approaches, in order to profit the advantages of each one of them. During the discovery, the proactive approach reduces the delay and increases the overhead due to the periodic broadcasting of control message; however, the reactive approach generates more delay and minimizes the overhead because the route discovery is executed only on demand. The illustration of the used hybrid approach is given to Fig. 6. 


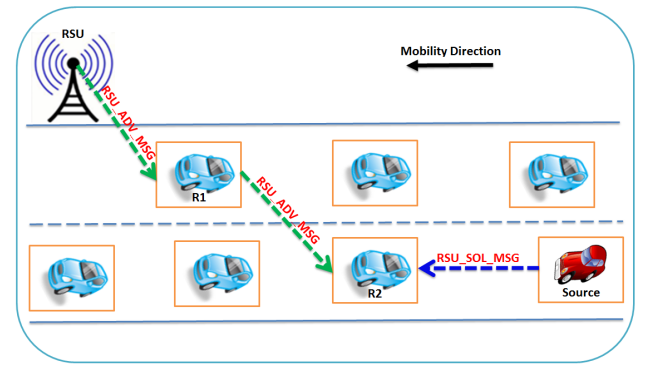

Fig. 6. Hybrid Route Discovery Approach

\section{F. Handover Process}

Handover is the process required to transfer the network connectivity of a vehicle from one RSU to another. Due to the high speed of vehicles, frequent disconnections in the network may happen. Connections should be seamlessly handed over to the next RSU before the current connection terminates. The more stables routes are always selected by vehicles to establish the connection over. In order to keep the connection, when the critical time is reached, vehicles must look up the routing table, to find the most stable route available to the next RSU, and start to transfer connection to that. The critical time $\left(T_{c}\right)$, means at what time vehicles decide that the current route is about to expire and the time has come to start the process of handover. It is defined as the effective route operation duration subtracted by the delay $T_{d}$ experienced by the last packet which has arrived along the route [10]. The expression of critical time is as follows :

$$
T_{c}=E R O D-T_{d}
$$

Here we have replaced route expiration time (RET) used in [9] with $E R O D$. By using the latest arrived packet to calculate $T_{c}$, the scheme is adaptive to changing network conditions and the vehicle will correctly take action in a timely manner.

\section{Simulation AND Results}

In this section, we discussed the performances of our proposed approach. To validate the proposed approach, we have simulated it and compare it with two approaches developed recently, in [10] MBRP (Mobility Based Routing Protocol), which based only on mobility vehicles parameters to measure link stability and M-AODV+ [6]. The proposed approach is noted MFBRP (Mobility and Fading Based Routing Protocol). We have performed some simulations in order to evaluate our proposed approach in term of throughput, the end to end packet delay and overhead by varying the maximum speed of vehicles and the number of vehicles on the road.

\section{A. Simulation Parameters}

In the current paper, we have based on the same simulation environment in the paper [11]. As illustrated in Fig. 7, our scenario is a highway of $8 \mathrm{Km}$ with two lanes, using simulators NS2 [24], MOVE [25], and SUMO [26]. All vehicles move from the one end of the highway to other end in the same direction and 10 vehicles are selected randomly to send CBR data at rate 20 packets/s to a node that is part of the wired network and is connected to all the base stations which are

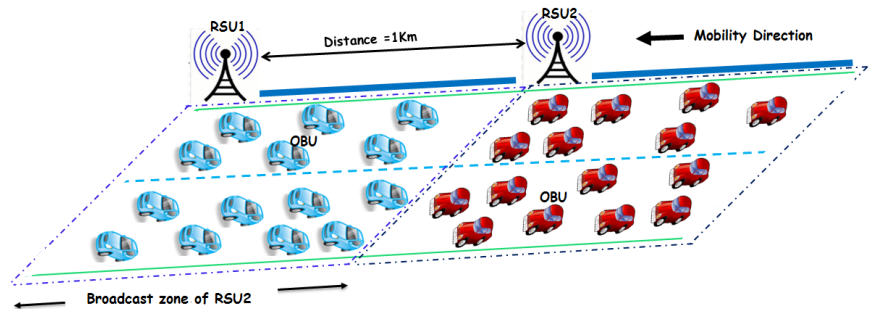

Fig. 7. Sample of our VANET scenario

TABLE II. NETWORK AND MOBility Parameters IN THE Simulation[11]

\begin{tabular}{|c|c|}
\hline Paramtre & Value \\
\hline Mobility model & Highway \\
\hline Highway length & $8 \mathrm{~km}$ \\
\hline Number of lanes & 2 \\
\hline Maximum speed & $10,20,30,40 \mathrm{~m} / \mathrm{s}$ \\
\hline Number of vehicles & $100,150,200,250$ \\
\hline Number of RSUs & 8 \\
\hline Distance between RSUs & $1000 \mathrm{~m}$ \\
\hline Simulation time & $460 \mathrm{~s}$ \\
\hline Pause time & $100 \mathrm{~s}$ \\
\hline Channel & Channel/WirelessChannel \\
\hline Propagation model & Nagakami $(m=3)$ \\
\hline Network Interface & Phy/WirelessPhyExt \\
\hline MAC & Mac802_11Ext \\
\hline Interface queue & QueueDropTailPriQueue \\
\hline Antenna Type & AntennaOmniAntenna \\
\hline Interface queue & 20 \\
\hline Transmission range & $300 \mathrm{~m}$ \\
\hline Routing protocols & M-AODV+ [6], MBRP [?] and MFBRP \\
\hline Addressing type & Hierarchical \\
\hline Traffic type & CBR \\
\hline Packet sending interval & $0.05 \mathrm{~s}$ \\
\hline Packet size & 512 bytes \\
\hline New parameters & $k=0.8, \lambda=E R O D / 2 \quad$ [27] \\
\hline$T_{\max }$ & $0,00375 \mathrm{~s} \quad[27]$ \\
\hline
\end{tabular}

connected to the wired network. To simulate protocols we have scheduled RSU to broadcast the advertisement message every $5 \mathrm{~s}$ in the predefined broadcast geographic zone which has been considered to be a circle with a radius of $1000 \mathrm{~m}$, and the message is broadcasted in the opposite movement of nodes. The TABLES II gives a summary of all simulation environment parameters.

M-AODV+ [6] is used in hybrid gateway discovery, its advertisement interval is fixed to $5 \mathrm{~s}$ and its advertisement zone is set to 3 hops, this means that an advertisement message will be only broadcast 3 times in the network, and nodes located further than 3 hops from a specific node have to send a route request message in order to find a route to that specific node.

\section{B. Simulation Result}

In this section, we present the analyses of the performance of our approach MBFRP in its both case (with and without $R S S$ ) in contrast with routing protocol proposed in [9] $M B R P$ and $M-A O D V+$.

1) Varying number of vehicles in the network: First, we compare the performance of the routing protocols by changing 


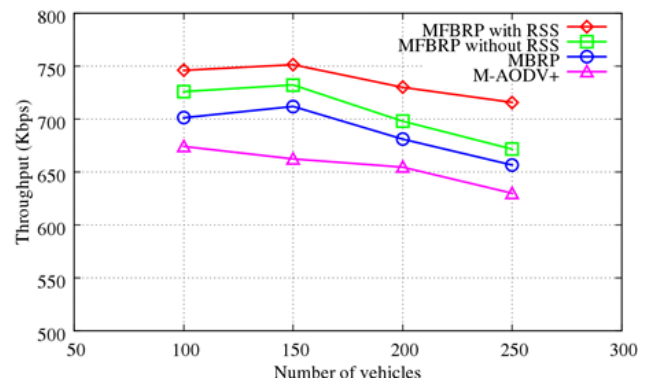

Fig. 8. Throughput comparison under different number of vehicles

the number of nodes in the network. The maximum speed of vehicles is fixed to $30 \mathrm{~m} / \mathrm{s}$ and the number of vehicular sources is fixed to 10 vehicles.

The simulation results for network throughput, average end-to-end delay and normalized routing control overhead, are shown in Fig. 8, Fig. 9 and Fig. 10 respectively. From the figures, it can be seen that while the network performance for all routing protocols decreased with the increased number of vehicles on the road. The performance degradation is due to the increased interference and congestion when the number of vehicles increases. As it is shown in the figures, the protocol MBRP outperforms M-AODV+. The reason behind this is that the former uses stability metric to select the most stable route, however, the later bases exclusively on the number of hops to choose a route and due to a large number of vehicles, the alternative wired communication overloads wired links between RSUs. We can see also, that our enhanced approach MFBRP has better performances in terms of throughput, average end-to-end delay and control overhead in the contrast of protocol MBRP. This is due to the considering the impact of fading statistics on the link stability because the fading channel introduces fast topology changes as well as mobility. ELOD and progress feature metrics using in MFBRP, makes the routing protocol to choose most stable and shortest route, to reduce network failure, increase network throughput and decrease end-to-end delay. As shown in the figures, MFBRP with RSS improves significantly network performance in contrast with MFBRP without RSS, this is because the former improves relay selection by considering the quality of received signal which is an important metric of quality of services. This metric leads the protocol to make able vehicles choose strongest routes, thus the packets will be arrived at the destination with enough reception power in order to be decodable. Consequently, the network performances, especially in term of packet loss, have been enhanced.

2) Varying maximum speed: We fixed the number of nodes at 200 vehicles and the number of vehicular sources at 10 sources, to evaluate the performance of the routing protocols with increasing maximum speed.

Fig. 11 illustrates the network throughput, while Fig. 12 shows the average end-to-end delay for the routing protocols and Fig. 13 depicts the normalized overhead routing with varying maximum speed. For all of the routing protocols, performances decrease with increasing vehicle mobility. The MFBRP always outperforms routing protocol MBRP and M-AODV+. The routing protocol M-AODV+ has the worst performance, although the changes that have been to support communication

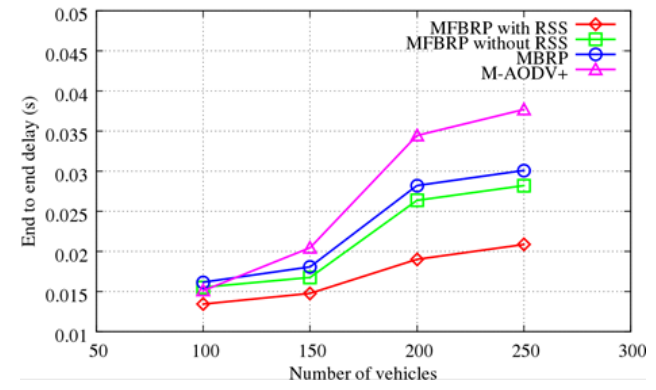

Fig. 9. Average end to end delay comparison under different number of vehicles

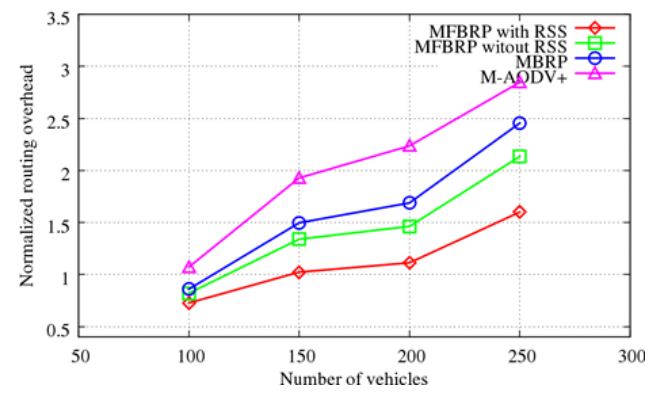

Fig. 10. Normalized overhead routing comparison under different number of vehicles

in VANETs. This is because M-AODV+ considers only hop count metric as main route selection parameters. The routing protocol MBRP has the good performance in contrast of M$\mathrm{AODV}+$. However, it is less efficient than MFBRP, which is because MBRP selects paths composed of links with longer lifetimes, but the paths might include more fading. The fading increases packets loss in the network and causes frequent topology change which will increase the cost in term of packet loss and control overhead routing, consequently, this will affect the network performances. As shown in the figures, the MFBRP with RSS has significant improvement of network performance in contrast with MFBRP without RSS. This is due that both approaches take account of link reliability and hopcount, but in addition to these both metrics, MFBRP with RSS incorporates an important route selection parameter of Quality of Service in mobile ad hoc network which is the quality of received signal. The reason behind this enhancement is because of the selective processing of signals. Only received signals which have RSS values exceed fixed thresholds, will be treated at the routing layer, this improves significantly the network performance especially in term of throughput and end to end delay. Note in our approach, we assume that the vehicles do not move during the interval in which the channel statistics are predicted, which might introduce some prediction errors and reduce the network performance, especially when the vehicle movement is high.

\section{CONCLUSION AND FUtURE WORKS}

VANET networks will play an important role in the future, and communication with road infrastructure needs to be covered to provide specific services such as Internet access. In this paper, we proposed an improving relay selection mechanism by considering two main metrics of quality of service which are link stability and quality of received signal in order to 


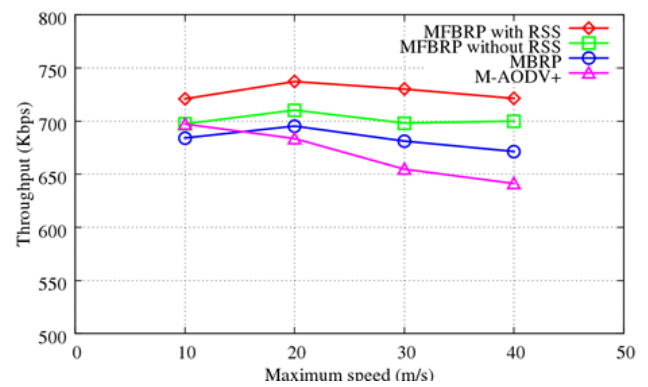

Fig. 11. Throughput comparison under different maximum speed

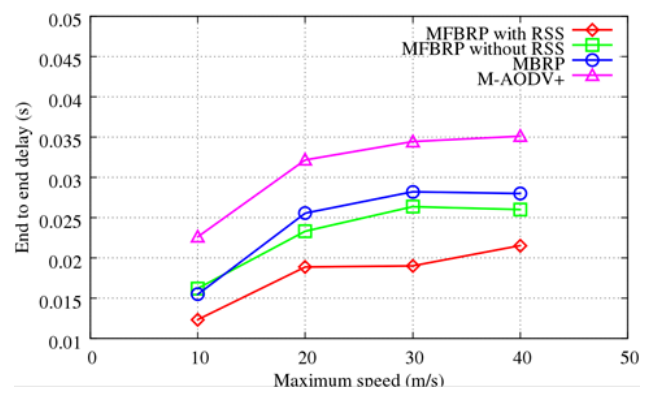

Fig. 12. Average end to end delay comparison under different maximum speed

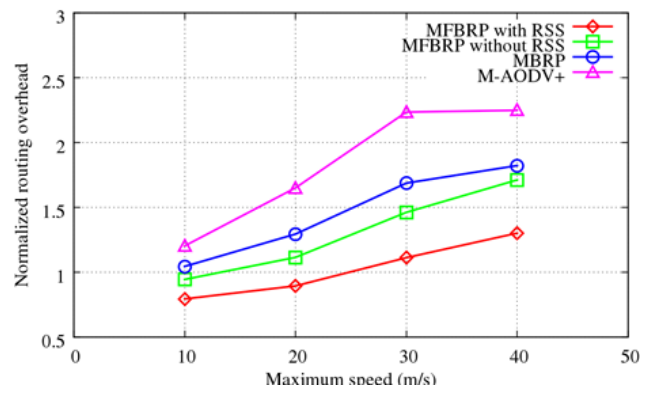

Fig. 13. Normalized overhead routing comparison under different maximum speed

select most stable and robust routes from vehicles to RSUs. Link stability is measured basing not only on the parameters of mobility but also on the effect of channel fading. This results in better performance and helps to maintain the good quality of the network. Simulation results show that our enhancing relay selection scheme achieves better performance than routing protocol MBRP and M-AODV+ over a range of network performance measures.

As the perspectives of the presented work, we will evaluate our approach in more realistic scenarios, especially in the urban area with high vehicle density and buildings. Even if IEEE $802.11 \mathrm{p}$ is a powerful and reliable protocol in VANET communications, there are still several issues which need to study IEEE $802.11 \mathrm{p}$ exhaustively in the context of V2V and V2I such as channel access, channel interferences, quality of service requirements, multichannel operation, exposed and hidden node effects, etc. We think in the future, to improve IEEE $802.11 \mathrm{p}$ to provide entertainment applications especially Internet access.

\section{REFERENCES}

[1] P.D. Dorge, D. .S. Dorle, M. B. Chakole, "Implementation of MIMO and AMC Techniques in WiMAX Network based VANET System," I.J. Information Technology and Computer Science,2016, pp. 60-68.

[2] B. Akbil, D. Aboutajdine, "Improved IDMA for Multiple Access of 5G," International Journal of Communication Networks and Information Security (IJCNIS), 2015, pp. 138-146.

[3] G. Araniti, C. Campolo, M. Condoluci, A. Iera, A. Molinaro, "LTE for Vehicular Networking: A Survey," IEEE Communications Magazine, 2013, pp. 148-157.

[4] Hameed Mir, Fethi Filali, "LTE and IEEE 802.11p for vehicular networking: a performance evaluation," EURASIP Journal on Wireless Communications and Networking, 2014.

[5] V.Jayaraj, C.Hemanth, R.G.Sangeetha, "A survey on hybrid MAC protocols for vehicular ad-hoc networks," Elsevier, 2016.

[6] J. Wantoro, I. W. Mustika, "M-AODV+: An Extension of AODV+ Routing Protocol for Supporting Vehicle-to-Vehicle Communication in Vehicular Ad hoc Networks," Communication, Networks and Satellite (COMNETSAT), IEEE International Conference, 2014, pp. 39-44.

[7] A. Benslimane, T. Taleb, R. Sivaraj, "Dynamic Clustering-Based Adaptive Mobile Gateway Management in Integrated VANET 3G Heterogeneous Wireless Networks," IEEE Journal on Selected Areas in Communications, 2011, pp. 559-570.

[8] A.A. Ba, A. Hafid, J. Drissi, "Broadcast Control-Based Routing Protocol for Internet Access in VANETS," International Wireless Communications and Mobile Computing, 2011.

[9] F. J. Ros, P. M. Ruiz, "Efficient Gateway Discovery Algorithms for Delay-tolerant and Delay-constrained Data Traffic in Vehicular Ad-hoc Networks," IEEE Vehicular Technology Conference, pp. 709-714, 2010.

[10] A. Benslimane, S. Barghi, C. Assi, An Efficient Routing Protocol for Connecting Vehicular Networks to the Internet, Pervasive and Mobile Computing Journal, Elsevier publisher, 2010.

[11] D. Abada, A. Massaq, A. Boulouz, "Enhacing relay selection scheme for connecting VANETs to Internet over IEEE 802.11p in Congested and Fading Environment Scenarios," International Review on Computers and Software (IRECOS), 2016, pp.410 - 419.

[12] A.Iera, A.Molinaro, S.Polito, and G. Ruggeri, "A MULTILAYER COOPERATION FRAMEWORK FOR QOS AWARE INTERNET ACCESS IN VANETS," Ubiquitous Computing and Communication Journal, 2008.

[13] M. Killat, H. Hartenstein, "An Empirical Model for Probability of Packet Reception in Vehicular Ad Hoc Networks," EURASIP Journal on Wireless Communications and Networking, 2008, pp. 1-12.

[14] A. Khan, S. Sadhu, and M. Yeleswarapu, "A comparative analysis of DSRC and 802.11 over Vehicular Ad hoc Networks," Project Report, Department of Computer Science, University of California, 2009, pp. $1-8$.

[15] S. Chen, H. Jones, D. Jayalath, "Effective link operation duration: a new routing metric for mobile ad hoc networks," International Conference on Signal Processing and Communication Systems,ICSPCS, 2007.

[16] K. B. BALTZIS, "On the Effect of Channel Impairments on VANETs Performance," RADIO ENGINEERING, 2010, pp. 689-694.

[17] H. Touil, Y. Fakhri, "A Fuzzy-based QoS Maximization Protocol for WiFi Multimedia (IEEE 802.11e) Ad hoc Networks," International Journal of Communication Networks and Information Security (IJCNIS), 2014, pp. 217-225.

[18] C. WU, S. OHZAHATA, T. KATO, "VANET Broadcast Protocol Based on Fuzzy Logic and Lightweight Retransmission Mechanism," IEICE TRANS COMMUNICATION, 2012.

[19] M. Asgari, M. Ismail, R.A. Alsaqour, "Mobility-aware Contentionbased Forwarding in Highway Vehicular Ad Hoc Networks," International Journal of Applied Engineering Research, 2015.

[20] F. Hrizi, C. Bonnet, J. Hrri, F. Filali, "Adapting Contention-Based Forwarding to Urban Vehicular Topologies for Active Safety Applications," Annals of telecommunications, Springer, 2013 , pp. 267-285.

[21] M. Asgari, M. Ismail, Raed Alsaqour, "Reliable Recovery Strategy for Contention-based Forwarding in Vehicular Ad hoc Network Streets," ARPN Journal of Engineering and Applied Sciences, 2015, pp. 91979207. 
[22] H. Sadeghian, A. Farahani, M.Abbaspour, "Overhead-controlled contention-based routing for VANETs," International Journal of Communication Networks and Information Security (IJCNIS), 2014, pp. 118-128.

[23] Y. Wang, A. Ahmed, B. Krishnamachari, K.Psounis, "IEEE 802.11p Performance Evaluation and Protocol Enhancement," IEEE International Conference on Vehicular Electronics and Safety, 2008, pp. 317332.

[24] The Network Simulator NS2, http://www.isi.edu/nsnam/ns/.

[25] F.K. Karnadi, Z.H. Mo, K.C Lan, "Rapid generation of realistic simulation for vanet ," IEEE WCNC, 2007.

[26] "Simulation of Urban Mobility," http://sumo.sourceforge.net.

[27] S. Barghi, A. Benslimane, C. Assi, "A lifetime-based routing protocol for connecting VANETs to the internet," in: WOWMOM, 2009, pp. 19. 